\title{
Método para a espacialização dos elementos do balanço hídrico climatológico
}

\author{
Roberto Avelino Cecílio(1), Kennedy Ribeiro da Silva(1), Alexandre Cândido Xavier ${ }^{(1)}$ \\ e José Ricardo Macedo Pezzopane ${ }^{(2)}$
}

\begin{abstract}
(1)Universidade Federal do Espírito Santo, Centro de Ciências Agrárias , Alto Universitário, s/no, Caixa Postal 16, CEP 29500-000 Alegre, ES. E-mail: rcecilio@cca.ufes.br, kennedyfloresta03@hotmail.com, xavier@cca.ufes.br ${ }^{(2)}$ Embrapa Pecuária Sudeste, Rodovia Washington Luiz, Km 234, Fazenda Canchim, CEP 13560-970 São Carlos, SP. E-mail: ricardo.pezzopane@gmail.com
\end{abstract}

Resumo - O objetivo deste trabalho foi desenvolver e avaliar um método de espacialização dos elementos do balanço hídrico climatológico (BHC). O método utiliza modelos digitais do terreno que contêm a distribuição espacial dos elementos climáticos de entrada do BHC. A avaliação foi feita com dados reais de BHC referentes a 110 estações pluviométricas do Estado do Espírito Santo. Comparou-se o desempenho do método proposto com o dos seguintes interpoladores espaciais: krigagem e inverso de uma potência da distância. O método proposto apresentou melhores estimativas espaciais de todos os elementos do balanço hídrico - evapotranspiração real, excedente, deficiência e disponibilidade hídrica.

Termos para indexação: deficiência hídrica, excedente hídrico, interpolação, krigagem.

\section{Method for spatialization of the climatic water balance elements}

\begin{abstract}
The objective of this work was to develop and evaluate a method for the spatial distribution of the climatic water balance (CWB) elements. The method applies terrain digital models containing the spatial distribution of climate elements used as input data of CWB. Evaluation was made with existing CWB data from 110 pluviometric stations of Espírito Santo state, Brazil. The proposed method's performance was compared to the one of the following spatial interpolators: kriging and inverse distance weighting. The proposed method presented best predictions of the spatial distribution of all CWB elements - actual evapotranspiration, water deficit, water surplus and water availability.
\end{abstract}

Index terms: water deficit, water surplus, interpolation, kriging.

\section{Introdução}

A produção agrícola, entre todas as atividades econômicas, é aquela que apresenta maior dependência das condições ambientais, em especial as climáticas (Pereira et al., 2002), principalmente no que diz respeito à disponibilidade de água. $\mathrm{O}$ primeiro passo para a obtenção de informações, quanto à disponibilidade potencial de água para os cultivos agrícolas, consiste no cálculo do balanço hídrico climatológico (BHC), que é a contabilização das entradas e saídas de água em um volume de solo vegetado, resultante do princípio de conservação de massa (Pereira et al., 2002). O BHC tem como elementos de saída as informações sobre evapotranspiração real (ETR), excedente hídrico (EXC), deficit hídrico (DEF) e disponibilidade hídrica (ER/ETP).

O conhecimento dos elementos do BHC norteia a gestão e o planejamento hidroagrícola, e subsidia: o zoneamento climático e agroecológico; a definição de épocas mais apropriadas de tratos culturais, como preparo do solo, semeadura e plantio, pulverização, colheita; a estimativa da produtividade de cultivos; o projeto e manejo da irrigação; o gerenciamento dos recursos hídricos em bacias hidrográficas; e a seleção e dimensionamento de técnicas voltadas à conservação do solo e da água (Pereira et al., 2002; Black, 2007; Sentelhas et al., 2008).

O balanço hídrico climatológico, normalmente, é calculado de forma pontual para estações em que há disponibilidade de dados climáticos. Posteriormente, com o emprego de técnicas de interpolação espacial, realiza-se a espacialização dos elementos de saída do BHC, a fim se ter uma representação de sua distribuição em grandes áreas, em um procedimento denominado "cálculo-interpolação". Alguns estudos têm utilizado diferentes métodos de interpolação espacial para estimativas geograficamente distribuídas de elementos

Pesq. agropec. bras., Brasília, v.47, n.4, p.478-488, abr. 2012 
do BHC. Todavia, alguns dos autores desses trabalhos não têm atentado para a necessidade de se avaliar qual o melhor método de interpolação (Lemos Filho et al., 2007; Sentelhas et al., 2008), e outros, embora mostrem tal preocupação, não encontraram métodos eficientes para a interpolação desses elementos, principalmente da deficiência e do excedente hídrico (Castro et al., 2010a).

Para processos não lineares, a estimação espacial dos elementos de interesse deve ser feita a partir da espacialização das variáveis de entrada necessárias e posterior cálculo espacial dos elementos de saída, procedimento denominado interpolação-cálculo. Tal procedimento tem grande potencial para ser aplicado na estimação espacial dos elementos do balanço hídrico, pois a variação de sua distribuição espacial também é não linear (Bechini et al., 2000).

O objetivo deste trabalho foi desenvolver e avaliar um método de espacialização dos elementos do balanço hídrico climatológico, baseado no princípio interpolação-cálculo, a partir do uso de modelos digitais do terreno com a distribuição espacial dos seguintes elementos de entrada: chuva e evapotranspiração potencial.

\section{Material e Métodos}

O método contemplou o Estado do Espírito Santo, entre as coordenadas $39^{\circ} 38^{\prime}$ e $41^{\circ} 50^{\prime} \mathrm{S}$ e $17^{\circ} 52^{\prime}$ e $21^{\circ} 19^{\prime} \mathrm{W}$, com área de aproximadamente $45.597 \mathrm{~km}^{2}$. Utilizaram-se dados climáticos medidos em 110 diferentes postos meteorológicos ou pluviométricos (Figura 1 A), localizados no Espírito Santo e estados adjacentes, com séries históricas de precipitação pluvial, em escala mensal, para o período de 30 anos. Os valores das temperaturas médias mensais do ar, que estavam indisponíveis nos postos, foram estimados pelas equações propostas por Castro et al. (2010b).

Desenvolveu-se, com o software MATLAB (MathWorks, 2010), um método para calcular os elementos do BHC de forma espacial, a partir de matrizes numéricas que continham a distribuição espacial mensal dos elementos do clima (precipitação e temperatura média do ar), conforme fluxograma na Figura 2. O BHC foi calculado conforme preconizado pelo método de Thornthwaite \& Mather(1955)(descrito em Pereira et al., 2002). Considerou-se a capacidade de água disponível no solo (CAD) igual a $100 \mathrm{~mm}$, valor comumente utilizado para culturas perenes e estudos de classificação climática. O procedimento básico do método para o cálculo do BHC consistiu da utilização de modelos digitais do terreno (MDT), no formato computacional do tipo raster, representativos da distribuição espacial mensal da precipitação $-\mathrm{P}_{\mathrm{x}, \mathrm{y}, \mathrm{i}}$ em que: $\mathrm{P}$ é a precipitação mensal (mm); x é a posição em longitude; y é a posição em latitude; e $\mathrm{i}=1,2, \ldots, 12$, os meses do ano; - e da temperatura média mensal do ar $\left(\mathrm{T}_{\mathrm{x}, \mathrm{y}, \mathrm{i}}\right)$ na área em estudo. Os dados de $\mathrm{P}$ e $\mathrm{T}$ foram espacializados com resolução espacial de aproximadamente $1 \mathrm{~km}$, o que resultou em matrizes de 266 colunas por 408 linhas para recobrir a área de estudo.

Para espacialização de $\mathrm{P}_{x, y, i}$ foram utilizados os dados mensais de chuva obtidos dos 110 postos pluviométricos anteriormente descritos. Os métodos de interpolação utilizados foram aqueles avaliados e recomendados por Silva et al. (2011). Os dados relativos a $T_{x, y, i}$ foram obtidos com as equações de regressão linear propostas por Castro et al. (2010b), em que T é dependente das coordenadas geográficas, latitude e longitude, e da elevação. Para a elevação, utilizaram-se os dados provenientes do projeto GTOPO30 (United States Geological Survey, 2012) (Figura 1 B).

A evapotranspiração potencial mensal em cada posição $\left(\mathrm{ETP}_{\mathrm{x}, \mathrm{y}, \mathrm{i}}\right)$ foi estimada a partir do emprego do método de Thornthwaite (1948), descrito em Pereira et al. (2002). A escolha de tal método se deveu ao fato de que este necessita apenas da temperatura do ar como dado meteorológico de entrada, além de apresentar apenas pequenos desvios de estimativa, em comparação ao método-padrão de Penman-Monteith, para o Estado do Espírito Santo, conforme trabalho de Sentelhas et al. (2008).

Os elementos do balanço hídrico calculados foram: evapotranspiração real anual, $\mathrm{ETR}_{\mathrm{x}, \mathrm{y}}=\Sigma \mathrm{ETR}_{\mathrm{x}, \mathrm{y}, \mathrm{i}}$; deficiência hídrica anual, $\mathrm{DEF}_{\mathrm{x}, \mathrm{y}}=\Sigma \mathrm{DEF}_{\mathrm{x}, \mathrm{y}, \mathrm{i}}$; excedente hídrico anual, $\mathrm{EXC}_{\mathrm{x}, \mathrm{y}}=\Sigma \mathrm{EXC}_{\mathrm{x}, \mathrm{y}, \mathrm{i}}$; e disponibilidade hídrica anual, $\mathrm{ETR}_{\mathrm{x}, \mathrm{y}} / \mathrm{ETP}_{\mathrm{x}, \mathrm{y}}=\Sigma \mathrm{ETR}_{\mathrm{x}, \mathrm{y}, \mathrm{i}} / \Sigma \mathrm{ETP}_{\mathrm{x}, \mathrm{y}, \mathrm{i}}$ (Figura 2).

A verificação do desempenho do método proposto foi realizada por meio de validação cruzada, que consiste retirar, isoladamente, uma das 110 estações e calcular o BHC a partir dos valores de $P_{i}$ e $E T P_{i}$ estimados para sua localização, para se obterem os valores estimados de ETR, DEF, EXC e ETR/ETP, que foram posteriormente comparados aos valores reais de cada variável. Estes valores reais foram obtidos por meio 
do cálculo pontual do BHC para os mesmos valores de CAD. Tal procedimento foi realizado separadamente para cada um dos postos localizados na área em estudos, com auxílio da rotina computacional de Xavier et al. (2010).

Utilizaram-se os seguintes índices estatísticos para o cálculo do desempenho: índice de confiança (c), coeficiente de eficiência ajustado (E') e erro médio percentual (EMP), apresentados nas equações a seguir. O índice de confiança (c) permite analisar conjuntamente a precisão e a exatidão dos resultados (Tabela 1)

$$
c=r\left[1-\frac{\sum_{i=1}^{J}\left(O_{i}-E_{i}\right)^{2}}{\sum_{i=1}^{J}\left(\left|E_{i}-\bar{O}\right|+\mid O_{i}-\bar{O}\right.}\right.
$$

$$
\begin{gathered}
E^{\prime}=1-\frac{\sum_{i=1}^{J}\left|O_{i}-E_{i}\right|}{\sum_{i=1}^{J}\left|O_{i}-\bar{O}\right|} \\
E M P=\frac{\sum_{i=1}^{J} \frac{\left|O_{i}-E_{i}\right|}{O_{i}}}{J} \times 100
\end{gathered}
$$

em que: $r$ é o coeficiente de determinação entre valores observados e estimados; J é o número de observações; O é o valor observado experimentalmente; E é o valor estimado pelo método; e $\overline{\mathrm{O}}$ é a média dos valores observados.
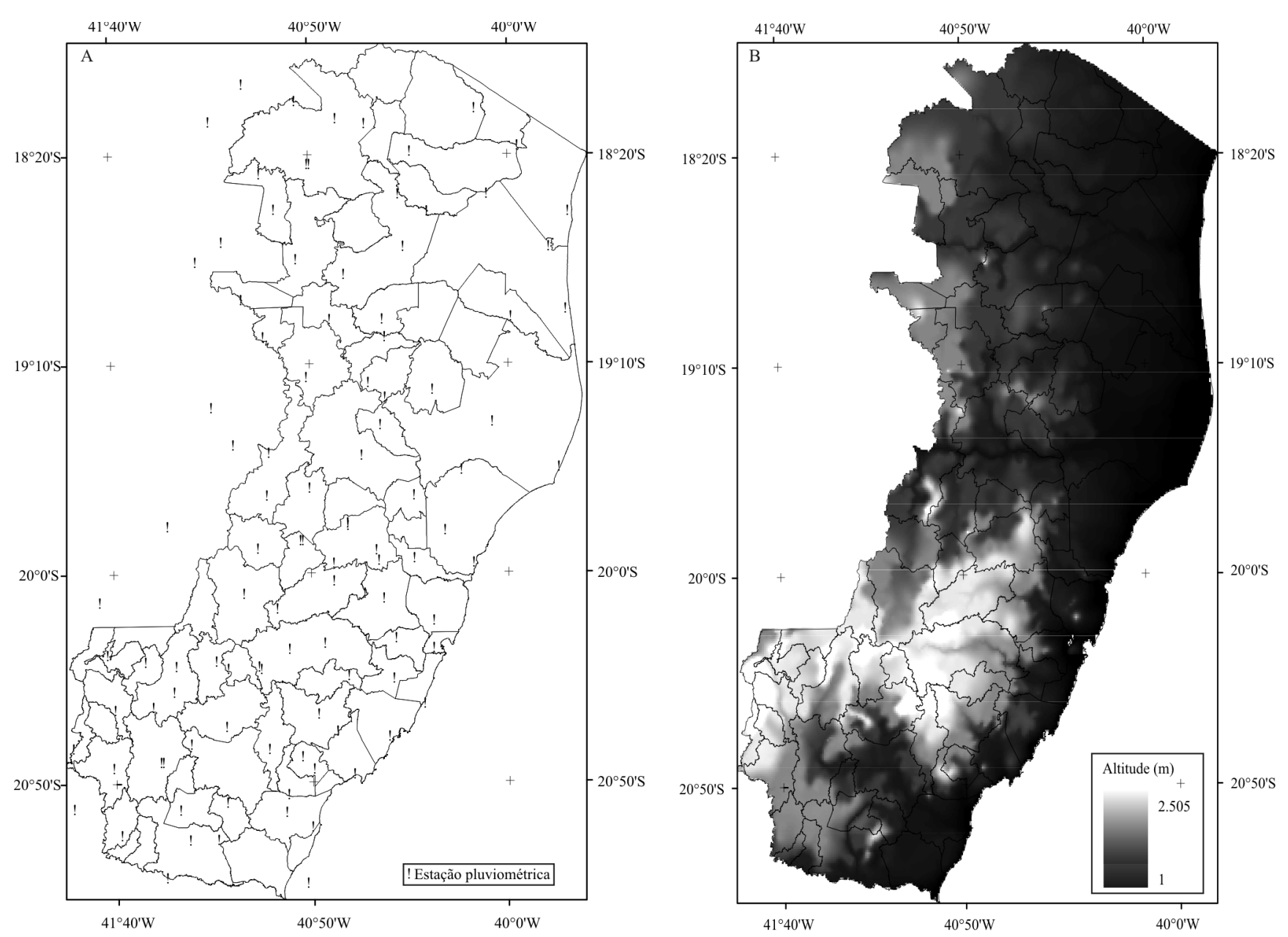

Figura 1. Estações pluviométricas utilizadas no estudo (A) e topografia do Estado do Espírito Santo (B). 
O desempenho do método proposto foi comparado ao de diferentes interpoladores, aplicados na espacialização dos elementos ETR, DEF, EXC e ETR/ ETP, obtidos de forma pontual a partir do cálculo do BHC para cada uma das estações localizadas na área em estudo. O desempenho dos interpoladores foi avaliado também com o uso do método de validação cruzada, tendo sido calculados os mesmos índices estatísticos anteriormente expostos. Os métodos de interpolação avaliados foram: inverso de potência da distância (IPD), com as potências de 1 a 6; e krigagem ordinária por meio dos modelos teóricos para semivariância do tipo linear, esférico, exponencial e gaussiano.

Para a aplicação da krigagem, foi realizada uma análise geoestatística, para quantificar o grau de dependência espacial dos dados, por meio de um semivariograma experimental estimado pela equação 4. Realizou-se o ajuste dos modelos teóricos (exponencial, esférico, gaussiano e linear) com patamar ao semivariograma experimental, de modo que a curva que melhor se ajustou aos pontos obtidos representa a magnitude, alcance e intensidade da variabilidade espacial da variável estudada.

$$
\gamma *(\mathrm{~h})=\frac{1}{2 \mathrm{~N}(\mathrm{~h})} \sum_{\mathrm{i}=1}^{\mathrm{N}(\mathrm{h})}\left[\mathrm{Z}\left(\mathrm{x}_{\mathrm{i}}\right)-\mathrm{Z}\left(\mathrm{x}_{\mathrm{i}}+\mathrm{h}\right)\right]^{2}
$$

em que: $\gamma^{*}(\mathrm{~h})$ é o valor do semivariograma estimado para a distância $h ; \mathrm{N}(\mathrm{h})$ é o número de pares de valores medidos; $\mathrm{x}_{\mathrm{i}}$ e $\mathrm{x}_{\mathrm{i}}+\mathrm{h}$ são os locais de amostragem, separados por uma distância $h ; Z\left(x_{i}\right)$ e $Z\left(x_{i}+h\right)$ são os valores medidos das variáveis nos locais correspondentes.

\section{Resultados e Discussão}

A análise apenas do desempenho dos interpoladores mostra que a krigagem, realizada por meio do modelo exponencial, se destaca por apresentar os melhores coeficientes estatísticos para todos os elementos do BHC (Tabela 2). Assim, os dados relativos aos interpoladores corroboram os resultados apresentados em Castro et al. (2010a) para a mesma área. De forma semelhante, Ribeiro (2009), ao trabalhar na parte norte da área em estudo, evidenciou superioridade da krigagem exponencial para interpolação espacial dos elementos ETR e ETR/ETP, em escala anual, embora

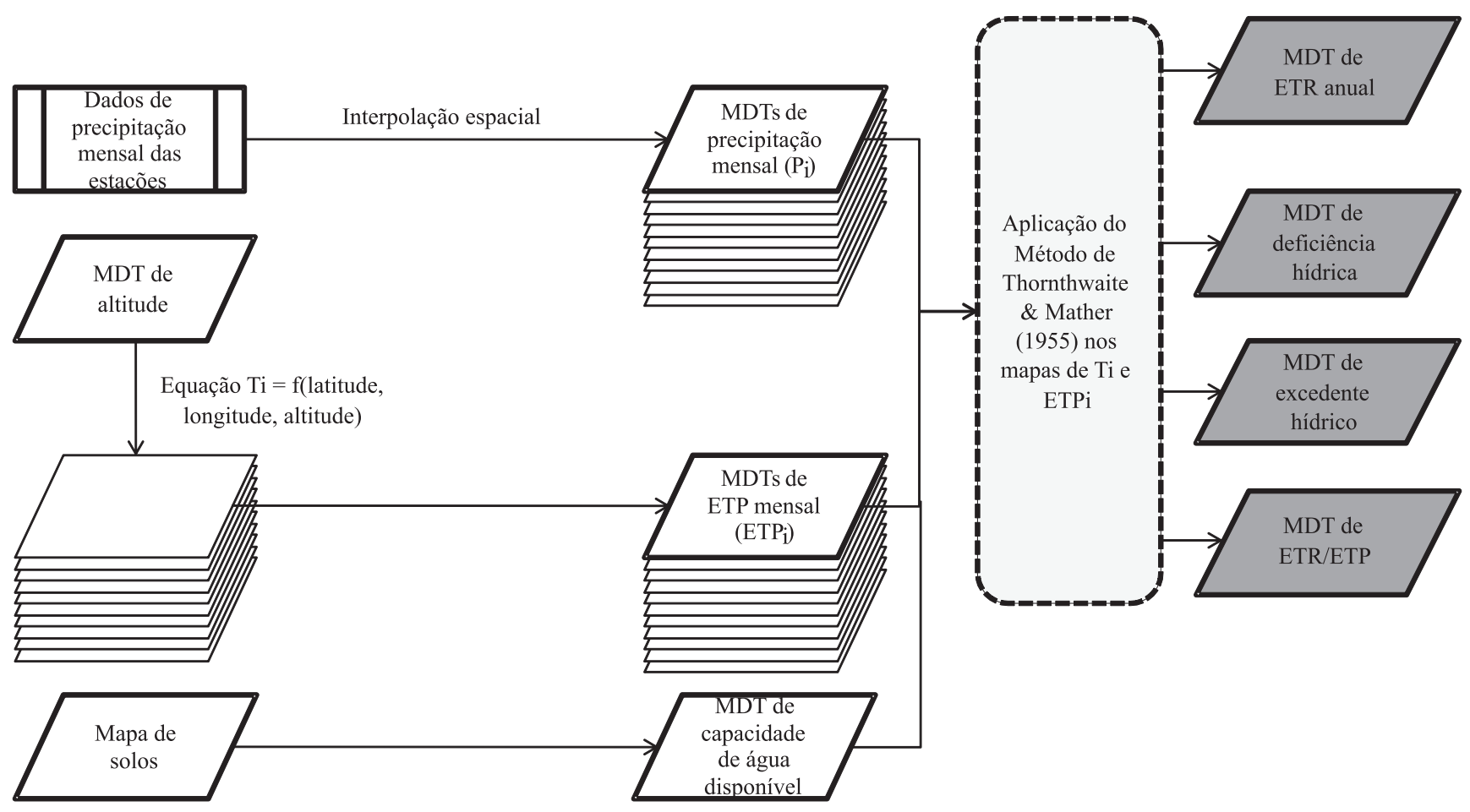

Figura 2. Fluxograma de etapas necessárias à aplicação do método proposto, para espacialização dos elementos do balanço hídrico pelo programa MATLAB (MathWorks, 2010). MDT, modelos digitais do terreno; ETP, evapotranspiração potencial mensal; ETR, evapotranspiração real; ETR/ETP, disponibilidade hídrica. 
os coeficientes de desempenho estatístico tenham sido inferiores aos do presente trabalho. A superioridade da krigagem sobre o IPD explica-se pelo fato de que a primeira é indicada para a aplicação em regiões onde a distribuição espacial dos dados observados é boa e sem descontinuidades significativas (Tabela 3). O IPD é indicado para locais com distribuição espacial boa e com alta densidade de estações (Akkala et al., 2010), o que não é o caso do presente trabalho. Além disso, a krigagem, a partir dos dados de entrada, determina os melhores parâmetros para modelagem da função matemática de interpolação, por meio do ajuste do

Tabela 1. Análise de desempenho de um modelo de espacialização dos elementos do balanço hídrico climatológico, com base no índice estatístico de confiança (c).

\begin{tabular}{lc}
\hline Valor de c & Desempenho \\
\hline$>0,85$ & Ótimo \\
0,76 a 0,85 & Muito bom \\
0,66 a 0,75 & Bom \\
0,61 a 0,65 & Mediano \\
0,51 a 0,60 & Sofrível \\
0,41 a 0,50 & Mau \\
$\leq 0,40$ & Péssimo \\
\hline
\end{tabular}

semivariograma, o que resulta em boas estimativas, quando os dados são bem representativos da região a ser modelada (Sivapragasam et al., 2010).

Embora em primeira análise o desempenho da krigagem exponencial tenha sido relativamente bom, com piores resultados para o elemento EXC (desempenho mediano), observa-se que os valores de EMP são demasiadamente altos para o EXC e o DEF (superiores a $1.000 \%$ ). Tal fato ocorre, em parte, em razão do grande número de valores muito próximos a zero, assumidos por esses elementos, que levam a altos erros percentuais.

Observa-se que os melhores desempenhos, na espacialização de todas as variáveis do $\mathrm{BHC}$, são obtidos quando da utilização do método proposto. Isso fica evidente na classificação igual ou superior a bom, nos altos valores de E' (superiores a 0,66), e nos baixos EMP (principalmente no que diz respeito ao DEF e EXC). Uma das causas do melhor desempenho é o fato de que o método proposto, diferentemente dos interpoladores, não permite a criação de uma superfície representativa dos elementos do BHC (ou MDT) que seja suavizada pelas funções matemáticas de interpolação, porque

Tabela 2. Índices estatísticos para análise de desempenho do método proposto e dos interpoladores, para a espacialização dos elementos evapotranspiração real, deficiência hídrica, excedente hídrico e disponibilidade hídrica, no Espírito Santo.

\begin{tabular}{|c|c|c|c|c|c|c|c|c|c|c|c|}
\hline \multirow[t]{2}{*}{ Índice estatístico } & \multicolumn{10}{|c|}{ Método de interpolação ${ }^{(1)}$} & \multirow[t]{2}{*}{ Método proposto } \\
\hline & K-EXP & K-ESF & K-GAU & K-LIN & IPD 1 & IPD 2 & IPD 3 & IPD 4 & IPD 5 & IPD 6 & \\
\hline \multicolumn{12}{|c|}{ Evapotranspiração real } \\
\hline $\mathrm{c}$ & 0,67 & 0,64 & 0,56 & 0,62 & 0,49 & 0,49 & 0,48 & 0,47 & 0,45 & 0,44 & 0,72 \\
\hline Desempenho & Bom & Mediano & Sofrível & Mediano & Mau & Mau & Mau & Mau & Mau & Mau & Bom \\
\hline $\mathrm{E}^{\prime}$ & 0,39 & 0,38 & 0,27 & 0,37 & 0,28 & 0,29 & 0,28 & 0,25 & 0,24 & 0,22 & 0,94 \\
\hline $\operatorname{EMP}(\%)$ & 6,3 & 6,5 & 7,3 & 6,6 & 7,7 & 7,6 & 7,7 & 8,0 & 8,2 & 8,3 & 6,2 \\
\hline \multicolumn{12}{|c|}{ Deficiência hídrica } \\
\hline c & 0,66 & 0,64 & 0,43 & 0,64 & 0,55 & 0,56 & 0,56 & 0,54 & 0,53 & 0,52 & 0,66 \\
\hline Desempenho & Bom & Mediano & Mau & Mediano & Sofrível & Sofrível & Sofrível & Sofrível & Sofrível & Sofrível & Bom \\
\hline$E^{\prime}$ & 0,43 & 0,42 & 0,25 & 0,41 & 0,32 & 0,34 & 0,34 & 0,33 & 0,31 & 0,29 & 0,72 \\
\hline $\operatorname{EMP}(\%)$ & $1.323,8$ & $1.344,1$ & $1.478,8$ & $1.321,5$ & $1.921,2$ & $1.936,2$ & $1.988,5$ & $2.078,9$ & $2.196,4$ & $2.326,0$ & 42,4 \\
\hline \multicolumn{12}{|c|}{ Excedente hídrico } \\
\hline $\mathrm{c}$ & 0,61 & 0,61 & 0,51 & 0,61 & 0,58 & 0,59 & 0,58 & 0,57 & 0,55 & 0,54 & 0,77 \\
\hline Desempenho & Mediano & Mediano & Sofrível & Mediano & Sofrível & Sofrível & Sofrível & Sofrível & Sofrível & Sofrível & Muito bom \\
\hline$E^{\prime}$ & 0,42 & 0,41 & 0,26 & 0,41 & 0,34 & 0,35 & 0,33 & 0,32 & 0,31 & 0,3 & 0,66 \\
\hline EMP (\%) & $8.691,7$ & $8.041,0$ & $10.710,6$ & $8.050,5$ & $18.775,6$ & $17.311,8$ & $16.090,6$ & $15.193,3$ & $14.552,1$ & $14.088,1$ & 58,1 \\
\hline \multicolumn{12}{|c|}{ Disponibilidade hídrica } \\
\hline $\mathrm{c}$ & 0,67 & 0,66 & 0,49 & 0,66 & 0,58 & 0,59 & 0,58 & 0,57 & 0,55 & 0,54 & 0,66 \\
\hline Desempenho & Bom & Bom & Mau & Bom & Sofrível & Sofrível & Sofrível & Sofrível & Sofrível & Sofrível & Bom \\
\hline $\mathrm{E}^{\prime}$ & 0,42 & 0,41 & 0,27 & 0,41 & 0,34 & 0,36 & 0,36 & 0,34 & 0,32 & 0,31 & 0,92 \\
\hline EMP (\%) & 5,4 & 5,5 & 6,7 & 5,5 & 6,2 & 6,1 & 6,1 & 6,3 & 6,4 & 6,6 & 7,9 \\
\hline
\end{tabular}

${ }^{(1)}$ K-EXP, krigagem exponencial; K-ESF, krigagem esférica; K-GAU, krigagem gaussiana; K-LIN, krigagem linear; IPD, inverso de uma potência da distância, em que os índices de 1 a 6 referem-se à potência utilizada; c, índice de confiança; E', coeficiente de eficiência ajustado; EMP, erro médio percentual. 
o BHC é calculado individualmente para cada célula (pixel) dos MDT. A krigagem, por sua vez, inserese em uma categoria de interpoladores que estima novos valores para os elementos interpolados nos pontos amostrais, por meio da função de ajuste do semivariograma, a fim se de criar uma superfície (ou MDT) suavizada (Falivene et al., 2010), de modo que os dados estimados para os pontos amostrais acabem por ser diferentes dos dados originais (Miranda et al., 2009). Outra causa é a utilização, pelo método proposto, de valores espacializados de ETP para estimativa dos elementos do balanço hídrico. Quando se utilizam interpoladores convencionais, a ETP sequer é considerada, pois apenas os resultados do BHC de cada posto são interpolados. Some-se a esse fato a utilização indireta da topografia, por meio da altitude, como uma variável auxiliar no processo de espacialização da variável climática ETP, a qual é sabidamente dependente da disponibilidade energética e da temperatura do ar (ambos influenciados pela topografia). A incorporação da topografia na espacialização é um procedimento recomendado quando a densidade de estações usadas é relativamente baixa e a distribuição espacial não é boa (Markidis et al., 2005), como no presente trabalho.

Nas espacializações realizadas, é possível verificar a influência da topografia nos MDT, quando se usa o método proposto e a suavização da superfície (MDT), quando da utilização do melhor dos interpoladores espaciais avaliados (krigagem exponencial), o que reforça a tendência anteriormente discutida de melhor desempenho da metodologia proposta (Figuras 3, 4, 5, 6).

Os valores de ETR (inferiores a $600 \mathrm{~mm}$ anuais), em sua maioria, ocorrem nas áreas mais altas do Estado, em que a temperatura e a disponibilidade

Tabela 3. Parâmetros dos modelos de semivariograma exponencial, ajustados para os modelos de krigagem, para estimativa espacial dos elementos do balanço hídrico no Espírito Santo

\begin{tabular}{lccc}
\hline Elemento $^{(1)}$ & ${\text { Efeito pepita }-\mathrm{c}_{0}}$ & ${\text { Variância estrutural }-\mathrm{c}_{1}}$ & Alcance $-\mathrm{a}\left({ }^{\circ}\right)$ \\
\hline ETR & $7.927,33$ & $19.305,35$ & 1,21 \\
DEF & $1.743,43$ & $16.189,14$ & 0,33 \\
EXC & $21.978,87$ & $13.753,92$ & 0,63 \\
ETR/ETP & 0,005 & 0,010 & 1,00 \\
\hline
\end{tabular}

(1)ETR, evapotranspiração real; DEF, deficiência hídrica; EXC, excedente hídrico; ETR/ETP, disponibilidade hídrica. de energia são menores, o que causa baixa ETP e, consequentemente, reduzidos valores de ETR (Figura 3). Isto pode ser comprovado pelos altos valores de ETR/ETP (superiores a 0,9) (Figura 6). A interpolação por krigagem não mostrou tais áreas, em razão da suavização propiciada pelo ajuste do semivariograma e da não contabilização da altitude na espacialização da ETP, conforme anteriormente discutido. De maneira geral, a tendência é de ocorrência dos maiores valores de ETR nas proximidades do litoral, com consequente redução em direção ao oeste do estado, o que denota influência marcante da longitude, oceanidade e, principalmente, do relevo.

Os mapas que contêm a espacialização do DEF mostram a tendência geral de menores valores nas maiores altitudes da região sul do estado, caracterizada por altos índices pluviométricos, resultantes da influência orográfica (Melo Júnior et al., 2006; Castro et al., 2010a), e menores temperaturas (Castro et al., 2010b) (Figura 4). Além disso, há tendência de maiores valores de DEF na porção centro-oeste do estado, caracterizada por baixa precipitação e elevadas temperaturas (Castro et al., 2010b). Mais uma vez, a influência da topografia é observada no MDT que contém a espacialização deste elemento por meio do método proposto, com o surgimento de uma classe de DEF superior a $400 \mathrm{~mm}$ anuais.

A distribuição espacial do elemento EXC mostra menores valores no norte do estado, de maior demanda evapotranspirativa em razão das maiores temperaturas do ar (Figura 5). Os maiores excedentes são verificados nas áreas mais elevadas da porção sul, em que se observam maiores precipitações (Castro et al., 2010a).

Quanto ao elemento ETR/ETP, os menores valores são verificados na porção centro-oeste do estado, justamente aquela com maiores valores de DEF, seguidos da porção norte (de elevadas temperaturas do ar); com maiores valores na porção sul do estado (Figura 6).

Convém enfatizar que, para uma boa espacialização dos elementos do BHC com o uso do método proposto, é fundamental que seja feita com critério a espacialização dos elementos do clima (precipitação e evapotranspiração potencial em escala mensal), usados como entrada de dados. 
Devem ser utilizados MDT de qualidade, isto é, bem representativos dos elementos do clima, conforme indicado por Bechini et al. (2000) e Loukas \& Vasiliades (2004).

No caso das precipitações mensais, recomendase que se utilizem dados provenientes de séries históricas relativamente longas (cerca de 30 anos ou mais), para que sejam efetivamente representativos do clima da região. Quando houver necessidade, indica-se que seja feita a análise de consistência e o preenchimento de falhas das séries históricas de chuva. Finalmente, que se avalie criteriosamente o método utilizado para espacialização dos dados mensais de chuva, nos moldes do que foi mostrado em diversos trabalhos da literatura especializada, com destaque, algumas vezes, para o IPD (Amorim et al., 2008; Ruelland et al., 2008), e frequentemente para a krigagem (Carvalho \& Assad, 2005; Siqueira et al., 2007; Ruelland et al., 2008; Silva et al., 2011) e técnicas geoestatísticas de cokrigagem com suporte da topografia (Hong et al., 2005; Viola et al., 2010) ou redes neurais artificiais (Sivapragasam et al., 2010). Outra opção seria a utilização de equações de regressão para a estimativa das precipitações em função das coordenadas locais e de altitude, conforme indicado por Naoum \& Tsanis (2003) e Mello \& Silva (2009). No presente trabalho, utilizaram-se métodos de interpolação espacial indicados por Silva et al.

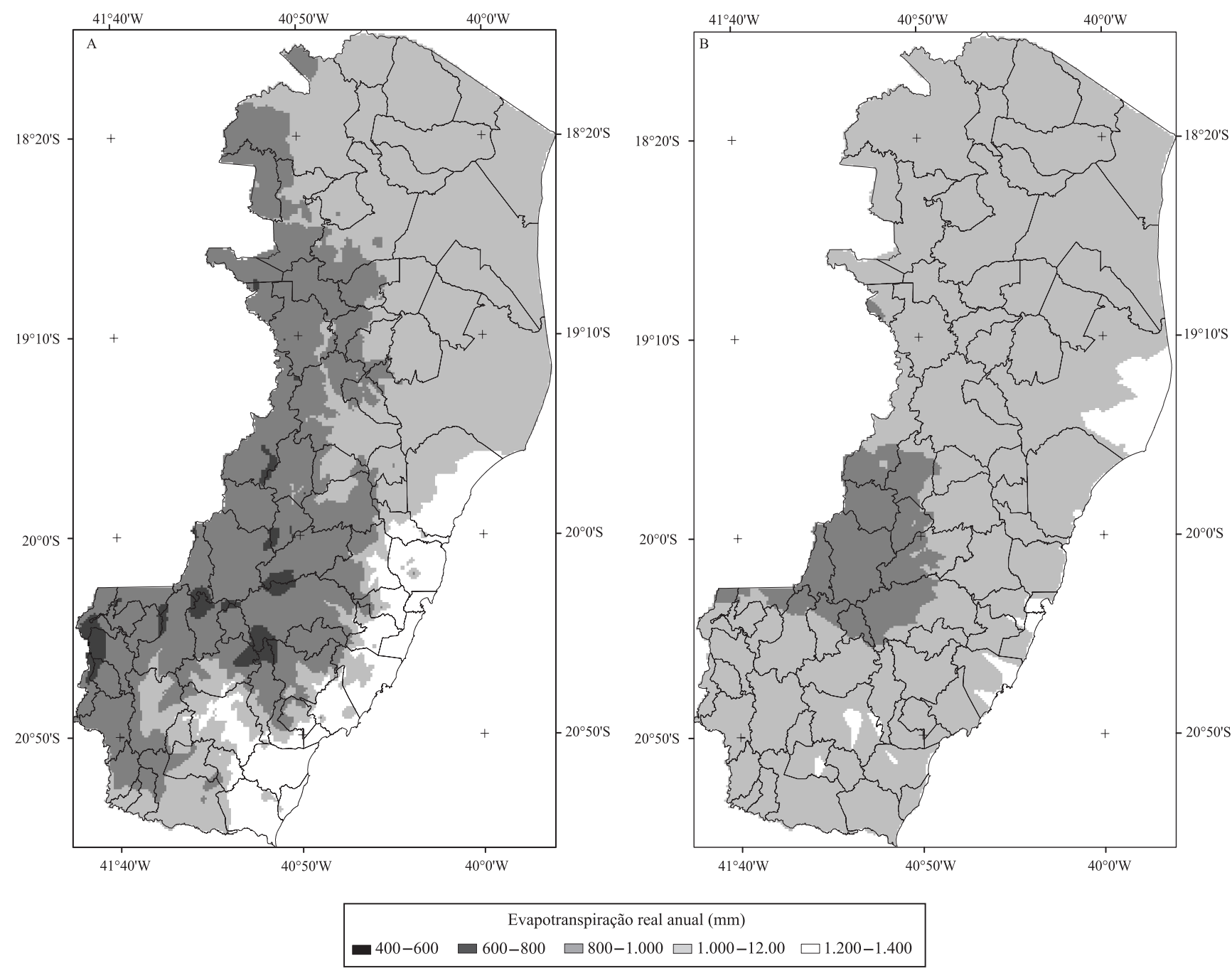

Figura 3. Espacialização da evapotranspiração real anual (ETR) no Estado do Espírito Santo, por meio do método proposto (A) e do interpolador krigagem exponencial (B). 
(2011), que fizeram um estudo criterioso de vários interpoladores para a área em estudo, indicados apenas para esta área. De forma alguma se recomenda a utilização dos mesmos interpoladores para outras regiões, sem que seja analisado seu desempenho, tarefa que vem se tornando cada vez mais fácil, pois hoje já existem ferramentas computacionais que agilizam o processo de análise de desempenho de interpoladores espaciais, como aquela desenvolvida por Xavier et al. (2010).

$\mathrm{Na}$ evapotranspiração média mensal, optou-se, no presente trabalho, em estimá-la por meio do método de Thornthwaite (1948), em virtude da baixa disponibilidade de dados e da comprovada eficiência deste método na área em estudo, comparativamente ao método-padrão de Penman-Monteith (Sentelhas et al., 2008). Nada impede que outros métodos de estimativa da ETP sejam usados, basta que sua seleção seja feita de forma criteriosa, evitando-se o uso de modelos não adaptados para as condições climáticas da área de estudos. Não se descarta a possibilidade de interpolação de dados de ETP originários de estações pontuais (Markidis et al., 2005; Lemos Filho et al., 2007; Castro et al., 2010a). Todavia, deve-se atentar para a análise da forma de interpolação desses dados, conforme discutido anteriormente para as precipitações. A espacialização

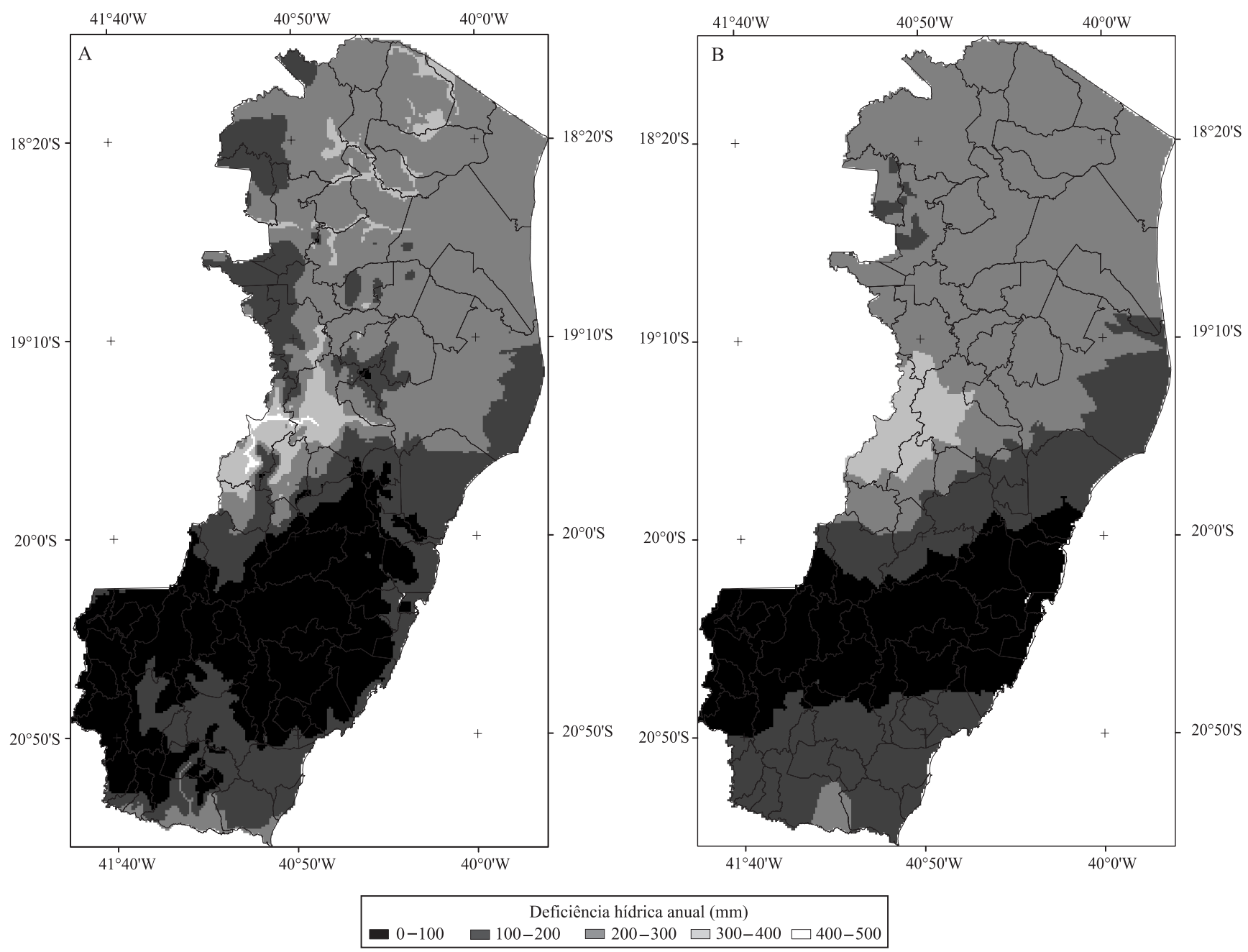

Figura 4. Espacialização da deficiência hídrica anual (DEF) no Estado do Espírito Santo, por meio do método proposto (A) e do interpolador krigagem exponencial (B). 
dos dados climáticos, para posterior estimativa da ETP, é recomendada (Sentelhas et al., 2008), uma vez que pode-se incluir o efeito da altitude em sua distribuição espacial, como foi feito com temperatura média do ar no presente trabalho. Os elementos do clima necessários para cálculo da ETP, a exemplo das temperaturas do ar, podem ser espacializados, a depender da situação, por meio de: métodos geoestatísticos de interpolação (Hong et al., 2005; Sentelhas et al., 2008; Mahdian et al., 2009); em função da localização geográfica e altitude; por meio de equações de regressão (Pilau et al., 2007; Castro et al., 2010b); ou, ainda, por redes neurais artificiais (Moreira \& Cecílio, 2008; Smith et al., 2009).

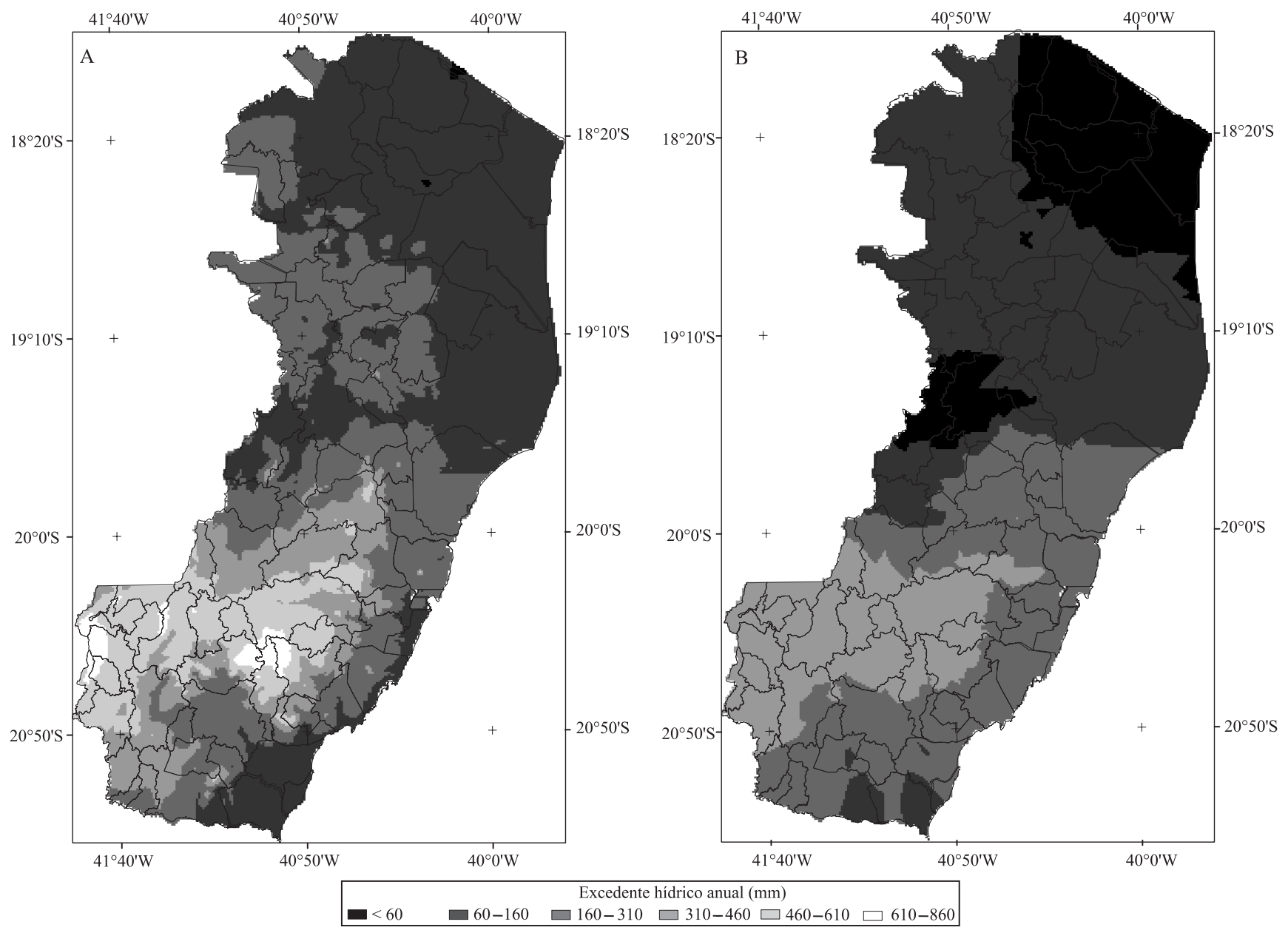

Figura 5. Espacialização do excedente hídrico anual (EXC) no Estado do Espírito Santo, por meio do método proposto (A) e do interpolador krigagem exponencial (B).
Finalmente, a capacidade de água disponível (CAD) é uma característica do solo essencial ao cálculo do BHC e varia de acordo com características inerentes ao próprio solo (textura, estrutura, densidade, porosidade, dentre outros) e com aquelas relacionadas à cultura de interesse (profundidade do sistema radicular). A definição de seu valor de forma incorreta altera os resultados do BHC, portanto, estudos de zoneamento climático de cultivos agrícolas podem ser prejudicados quando da consideração equivocada do valor assumido pela CAD. Uma alternativa potencial é considerar os valores da CAD, que variem em função do tipo de solo (Sperandio et al., 2010), o que pode ser incorporado no presente método por meio da 


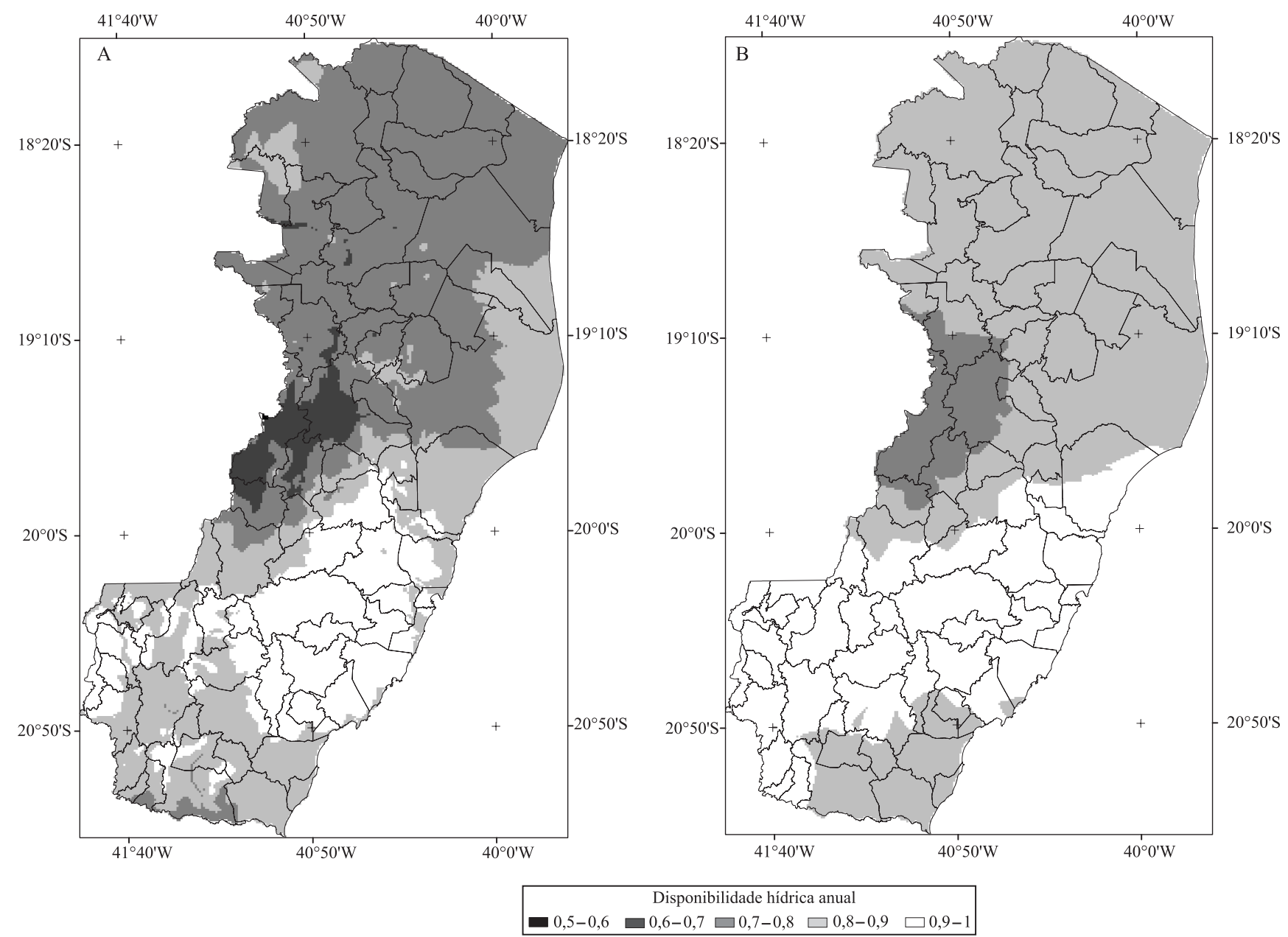

Figura 6. Espacialização da disponibilidade hídrica anual (ETR/ETP) no Estado do Espírito Santo, por meio do método proposto (A) e do interpolador krigagem exponencial (B).

espacialização da CAD, que poderia ser gerada por meio de uma ponderação, considerando-se o tipo do solo que é encontrado nos mapas de solos.

\section{Conclusão}

O método com uso de modelos digitais de terreno, que contém a distribuição espacial dos elementos climáticos, proposto para espacializar os elementos do balanço hídrico climatológico, apresenta desempenho superior ao dos interpoladores espaciais avaliados.

\section{Agradecimentos}

À Fundação de Amparo à Pesquisa do Espírito Santo e ao Conselho Nacional de Desenvolvimento Científico e Tecnológico, pela concessão de bolsas.

\section{Referências}

AKKALA, A.; DEVABHAKTUNI, V.; KUMAR, A. Interpolation techniques and associated software for environmental data. Environmental Progress and Sustainable Energy, v.29, p.134-141, 2010.

AMORIM, R.C.F. de; RIBEIRO, A.; LEITE, C.C.; LEAL, B.G.; SILVA, J.B.G. Avaliação do desempenho de dois métodos de espacialização da precipitação pluvial para o Estado de Alagoas. Acta Scientiarum. Technology, v.30, p.87-91, 2008.

BECHINI, L.; DUCCO, G.; DONATELLI, M.; STEIN, A. Modelling, interpolation and stochastic simulation in space and time of global solar radiation. Agriculture, Ecosystems and Environment, v.81, p.29-42, 2000.

BLACK, P. Revising the Thornthwaite and Mather water balance. Journal of the American Water Resources Association, v.43, p.1604-1605, 2007. 
CARVALHO, J.R.P. de; ASSAD, E.D. Análise espacial da precipitação pluviométrica no Estado de São Paulo: comparação de métodos de interpolação. Engenharia Agrícola, v.25, p.377-384, 2005.

CASTRO, F. da S.; PEZZOPANE, J.E.M.; CECÍLIO, R.A.; PEZZOPANE, J.R.M.; XAVIER, A.C. Avaliação do desempenho dos diferentes métodos de interpoladores para parâmetros do balanço hídrico climatológico. Revista Brasileira de Engenharia Agrícola e Ambiental, v.14, p.871-880, 2010a.

CASTRO,F.daS.;PEZZOPANE,J.R.M.;CECÍLIO,R.A.;PEZZOPANE, J.E.M. Uso de imagens de radar na espacialização da temperatura do ar. Idesia, v.28, p.69-79, 2010b.

FALIVENE, O.; CABRERA, L.; TOLOSANA-DELGADO, R.; SÁEZ, A. Interpolation algorithm ranking using cross-validation and the role of smoothing effect: a coal zone example. Computers and Geosciences, v.36, p.512-519, 2010.

HONG, Y.; NIX, H.; HUTCHINSON, M.; BOOTH, T. Spatial interpolation of monthly mean climate data for China. International Journal of Climatology, v.25, p.1369-1379, 2005.

LEMOS FILHO, L.C. de A.; CARVALHO, L.G.; EVANGELISTA, A.W.P.; CARVALHO, L.M.T.; DANTAS, A.A.A. Análise espaço-temporal da evapotranspiração de referência para Minas Gerais. Ciência e Agrotecnologia, v.31, p.1462-1469, 2007.

LOUKAS, A.; VASILIADES, L. Probabilistic analysis of drought spatiotemporal characteristics in Thessaly region, Greece. Natural Hazards and Earth System Sciences, v.4, p.719-731, 2004.

MAHDIAN, M.H.; BANDARABADY, S.R.; SOKOUTI, R.; BANIS, Y.N. Appraisal of the geostatistical methods to estimate monthly and annual temperature. Journal of Applied Sciences, v.9, p.128-134, 2009.

MARKIDIS, M.G.; KALIVAS, D.P.; KOLLIAS, V.J. Comparison of interpolation methods for the prediction of reference evapotranspiration - an application in Greece. Water Resources Management, v.19, p.251-278, 2005 .

MATHWORKS. MATLAB and Simulink: release notes for R2010. Natick: MathWorks, 2010.

MELLO, C.R. de; SILVA,A.M. da. Modelagem estatística da precipitação mensal e anual e no período seco para o Estado de Minas Gerais. Revista Brasileira de Engenharia Agrícola e Ambiental, v.13, p.68-74, 2009.

MELO JÚNIOR, J.C.F. de; SEDIYAMA, G.C.; FERREIRA, P.A.; LEAL, B.G. Determinação de regiões homogêneas quanto à distribuição de freqüência de chuvas no leste do Estado de Minas Gerais. Revista Brasileira de Engenharia Agrícola e Ambiental, v.10, p.408-416, 2006.

MIRANDA, F.A.; FREITAS, S.R.C. de; FAGGION, P.L. Integração e interpolação de dados de anomalias ar livre utilizando-se a técnica de RNA e krigagem. Boletim de Ciências Geodésicas, v.15, p.428-433, 2009.

MOREIRA, M.C.; CECÍLIO, R.A. Estimativa das temperaturas do ar utilizando redes neurais artificiais para a região Nordeste do Brasil. Revista Brasileira de Agrometeorologia, v.16, p.159-166, 2008.
NAOUM, S.; TSANIS, I.K. Temporal and spatial variation of annual rainfall on the island of Crete, Greece. Hydrological Processes, v.17, p.1899-1922, 2003.

PEREIRA, A.R.; ANGELOCCI, L.R.; SENTELHAS, P.C. Agrometeorologia: fundamentos e aplicações práticas. Guaíba: Agropecuária, 2002. 487p.

PILAU, G.F.; MARIN, F.R.; CAMARGO, M.B.P. de; ASSAD, E.D.; PINTO, H.S.; BARBARISI, B.F. Zoneamento agroclimático da heveicultura para as regiões Sudeste e Centro-Oeste do Brasil. Revista Brasileira de Agrometeorologia, v.15, p.161-168, 2007.

RIBEIRO, C.A.D. Delimitação de zonas agroclimáticas para cultura do eucalipto no norte do Espírito Santo e sul da Bahia. 2009. 104p. Dissertação (Mestrado) - Universidade Federal do Espírito Santo, Alegre.

RUELLAND, D.; ARDOIN-BARDIN, S.; BILLEN, G.; SERVAT, E. Sensitivity of a lumped and semi-distributed hydrological model to several methods of rainfall interpolation on a large basin in West Africa. Journal of Hydrology, v.361, p.96-117, 2008.

SENTELHAS, P.C.; SANTOS, D.L. dos; MACHADO, R.E. Water deficit and water surplus maps for Brazil, based on FAO Penman-Monteith potential evapotranspiration. Revista Ambiente e Água, v.3, p.28-42, 2008

SILVA, K.R. da; CECÍLIO, R.A.; XAVIER, A.C.; PEZZOPANE, J.R.M.; GARCIA, G. de O. Interpolação espacial da precipitação no Estado do Espírito Santo. Floresta e Ambiente, v.18, p.417-427, 2011.

SIQUEIRA, H.R. de; ALVES, G.F.; GUIMARÃES, E.C. Comportamento da precipitação pluviométrica mensal do Estado de Minas Gerais: análise espacial e temporal. Horizonte Científico, v.1, p.1-21, 2007.

SIVAPRAGASAM, C.; ARUN, V.; GIRIDHAR, D. A simple approach for improving spatial interpolation of rainfall using ANN. Meteorology and Atmospheric Physics, v.109, p.1-7, 2010.

SMITH, B.A.; HOOGENBOOM, G.; MCCLENDON, R.W. Artificial neural networks for automated year-round temperature next term prediction. Computers and Electronics in Agriculture, v.68, p.52-61, 2009.

SPERANDIO, H.V.; CAMPANHARO, W.A.; CECÍLIO, R.A.; NAPPO, M.E. Zoneamento agroecológico para espécies de eucalipto no Estado do Espírito Santo. Caminhos de Geografia, v.11, p.203-216, 2010.

UNITED STATES GEOLOGICAL SURVEY. GETOPO30. Available at: <http://eros.usgs.gov/\#/Find_Data/Products_and_Data_Available/ gtopo30 info $>$. Accessed on: 14 May 2012.

VIOLA, M.R.; MELLO, C.R. de; PINTO, D.B.F.; MELLO, J.M. de; ÁVILA, L.F. Métodos de interpolação espacial para o mapeamento da precipitação pluvial. Revista Brasileira de Engenharia Agrícola e Ambiental, v.14, p.970-978, 2010.

XAVIER, A.C.; CECÍLIO, R.A.; LIMA, J.S. de S. Módulos em MATLAB para interpolação espacial pelo método de krigagem ordinária e do inverso da distância. Revista Brasileira de Cartografia, v.62, p.67-76, 2010.

Recebido em 11 de março de 2011 e aprovado em 20 de março de 2012

Pesq. agropec. bras., Brasília, v.47, n.4, p.478-488, abr. 2012 